\title{
SUSCEPTIBILITY TO MITOMYCIN C AND LECITHINASE ACTIVITIES OF CLOSTRIDIUM OEDEMATIENS (C. NOVYI) TYPES B AND D
}

\author{
S. Nakamura, K. Takematsu and S. Nishida \\ Department of Bacteriology, School of Medicine, Kanazawa University, Kanazawa, Japan
}

\section{Plate XVI}

It is still technically difficult to distinguish some strains of Clostridium oedematiens (C. novyi) of types B and D (Rutter and Collee, 1969). Type-A strains of $C$. oedematiens produce a haemolytic lecithinase (the $\gamma$-toxin) which is distinct from the haemolytic lecithinase ( $\beta$-toxin) produced by type-B and type-D strains. As type-D strains are known to produce more of the $\beta$-toxin lecithinase than type-B strains, it was hoped to determine ranges of this lecithinase activity, here referred to as $\beta$-lecithinase, that would discriminate between the two types. In the course of studies on the lysogeny of clostridia (Kiritani et al., 1973; Shimamura et al., 1974), we found that type-D strains differed from typeB strains of $C$. oedematiens in their susceptibility to mitomycin C (MC). This was investigated further in the present study with a view to differentiating type-B and type-D strains. Since treatment of bacteria in the lysogenic state by $\mathrm{MC}$ induces multiplication of phage, and since lysogeny is sometimes related to bacterial toxigenicity, we also examined strains of $C$. oedematiens of types B and D in parallel for susceptibility to MC and for production of the $\beta$-lecithinase.

\section{MATERIALS AND METHODS}

\section{Bacterial strains}

C. oedematiens type A. Toxigenic strains $77101,77103,37101,37106$, and 37110 and non-toxigenic strains 3-1103, 3-1105, 3-1111, and 3-1112 are stock strains isolated from soil by one of the authors in 1963 and typed by Nishida and Nakagawara (1964). Strain 140 was kindly supplied in 1962 as a highly toxigenic strain by Dr M. Miyazaki, The Institute for Infectious Disease, Tokyo, Japan.

C. oedematiens type B. Strains 990,992 , and 2879 were kindly provided by Dr P. Høgh, the State Veterinary Serum Laboratory, Copenhagen, Denmark; strains GR1B, GR2B, GR3B, GR4B, GR7B, GR8B, GR11B, GR12B, and GR13B by Dr J. G. Collee, Department of Bacteriology, University Medical School, Edinburgh; strain 1787 by Dr Irene Batty, Wellcome Research Laboratories, England; and strain Kitamura by Dr R. Azuma, the Institute for Veterinary Hygiene Laboratory, Tokyo, Japan. Strain 25758 was obtained from the Anaerobe Laboratory, Virginia Polytechnic Institute and State University, Blacksburg, Virginia, USA.

C. oedematiens type $D$. Strains 8933, 9502, 2116, 2168, 6700, 3630, 6705, 6514, 2014, 4507, 9192, and 13135 were kindly provided by Dr P. Høgh; and strain Shinketsu by Dr R. Azuma. 
The typing of our stock strains of $C$. oedematiens types $B$ and $D$ was re-confirmed by mouse-lethality tests and by neutralisation tests with type-A and type-B antisera (Wellcome Research Laboratories, Beckenham, England).

Non-toxigenic strains of $C$. oedematiens type $B$ or $D$. The strains that did not produce any lethal toxin but produced a slight amount of $\beta$-lecithinase were designated as non-toxigenic strains of $C$. oedematiens type $B$ or $D$; these are strains Higumakan and Mizuno-81 provided by Dr R. Azuma, and NT (" non-toxic") provided by Dr P. Høgh.

\section{Media}

Tarozzi's liver broth was used as a preliminary culture medium in the present study; swine liver replaced guinea-pig liver in the preparation of this medium (Winkle, 1955). The egg-yolk-agar plate employed is a modified Weinberg's agar (Nagler, 1944). To test $\alpha$-toxigenicity, 72 -h-old cultures in Vf broth containing $0.5 \%$ glucose (VfG) was used. For the production of $\beta$-lecithinase, TYG medium $(\mathrm{pH} \mathrm{7.8)}$ consisting of trypticase (BBL) $3 \%$ $(w / v)$, yeast extract (Difco) $1 \%(w / v)$, glucose $0.5 \%, \mathrm{NaCl} 0.5 \%, \mathrm{Na}_{2} \mathrm{HPO}_{4} .12 \mathrm{H}_{2} \mathrm{O} 0.2 \%$, and sodium thioglycollate $0.1 \%$, was mainly used. As reference media for the production of $\beta$-lecithinase, we used VfG medium and the T-F medium $(p H \mathbf{H} \cdot 1)$ of Tamai and Fukuda (1970) which contained Brain-Heart Infusion (Nissan, Tokyo, Japan) $2.5 \%(w / v)$, yeast extract $0.3 \%$, glucose $0.5 \%$, and " modified TGC" (Nissan, Tokyo, Japan) $3.0 \%$ (w/v); the modified TGC consists of trypticase $17.0 \mathrm{~g}$, phytone $3.0 \mathrm{~g}$, glucose $6.0 \mathrm{~g}, \mathrm{NaCl} 2.5 \mathrm{~g}$, agar $0.7 \mathrm{~g}$, and sodium thioglycollate $0.5 \mathrm{~g}$ per litre.

Anaerobic jar procedure. Anaerobic jars with Deoxo pellets (Baker Platinum Ltd, London) as the catalyst were employed. Culture media were incubated as a routine at $37^{\circ} \mathrm{C}$ in an anaerobic hydrogen atmosphere.

\section{Biochemical tests}

The fermentation test procedure was as described by Rutter (1970). Carbohydrate solutions were prepared as $10 \%$ solutions and were sterilised by Seitz-filtration; an appropriate volume of each solution was added to the sterile basal medium to give a final concentration of $1 \%(w / v)$ of the added substrate.

Test for indole. The production of indole was tested with 24 - and 48-h cultures in cookedmeat broth containing tryptophan $1 \mu \mathrm{g}$ per $\mathrm{ml}$. Indole was extracted by the addition of $1 \mathrm{ml} x y l e n e$, and Ehrlich's reagent was then added.

Estimation of lecithinase activity. Cultures grown for 6-12 h in TYG medium were centrifuged and the supernates were examined for lecithinase activity according to the method of van Heyningen (1941) modified by Mitsui, Mitsui and Hase (1973), the results being expressed in egg-units (EU) per $\mathrm{ml}$. A glycerinated standard solution of $C$. perfringens lecithinase was kindly supplied by Dr K. Mitsui, Faculty of Pharmaceutical Science, University of Toyama, Japan; the potency of this preparation was calculated according to the equation, mouse LD50: $\mathrm{EU}=3: 1$.

\section{Studies of susceptibility to mitomycin $C(M C)$}

Volumes $(1 \mathrm{ml})$ of bacterial cultures grown in T-F medium for $16 \mathrm{~h}$ were seeded into 20 -ml volumes of T-F medium in tubes $(2 \times 15 \mathrm{~cm})$ and incubated at $37^{\circ} \mathrm{C}$ until the optical density reading at $560 \mathrm{~nm}\left(E_{560}\right)$ of the bacterial growth reached 0.15 to 0.2 . Then MC (Sankyo Co., Tokyo, Japan) was added in graded amounts to give final concentrations of $0.5,1.0,2.0$ or $5.0 \mu \mathrm{g}$ per $\mathrm{ml}$ of culture. The reaction mixtures containing culture samples with MC added were then incubated aerobically pending bacterial lysis. The effect of MC was recorded as positive when bacterial lysis sufficient to reduce the $E_{560}$ reading by at least 0.15 in the culture occurred within $6 \mathrm{~h}$ after the addition of the drug. Optical density was measured by a Shimazu-Bosch-Lomb Spectronic 20 Spectrophotometer (Shimazu Co., Kyoto, Japan) in tubes. Results were confirmed by repeating each experiment at least once. 
Detection of phage particles by electronmicroscopy

Preparation of samples and the procedures for electronmicroscopy were as described by Shimamura et al. (1974).

\section{RESULTS}

\section{Lecithinase studies}

$A$ modified in-vitro typing procedure. In preliminary attempts to use the plate method of Oakley, Warrack and Clarke (1947) to distinguish C. oedematiens types $B$ and D from type A with suitable antisera, we encountered considerable difficulties because strains of types B and D failed to grow as surface cultures on agar media (Rutter and Collee, 1969). The neutralisation test, therefore, was modified as follows. Strains of types B and D were cultured in VfG broth, which consistently supported their good growth. As shown in fig. 1, 0.3-ml portions of the $48-\mathrm{h}$ culture supernate were pipetted into wells in an agar plate supplemented with $5 \%$ egg yolk, and a 0.3 -ml portion of suitable antiserum was put into the central well. The plate was kept at $37^{\circ} \mathrm{C}$ for $48 \mathrm{~h}$ and results were then recorded. The culture products of all of 15 type-B, 13 type-D and 3 type-B or type-D strains exhibited wide lecithinase zones that were neutralised by the type-B but not by the type-A antiserum. All of the type-A culture products gave extremely narrow lecithinase zones that were covered by the characteristic pearly precipitate.

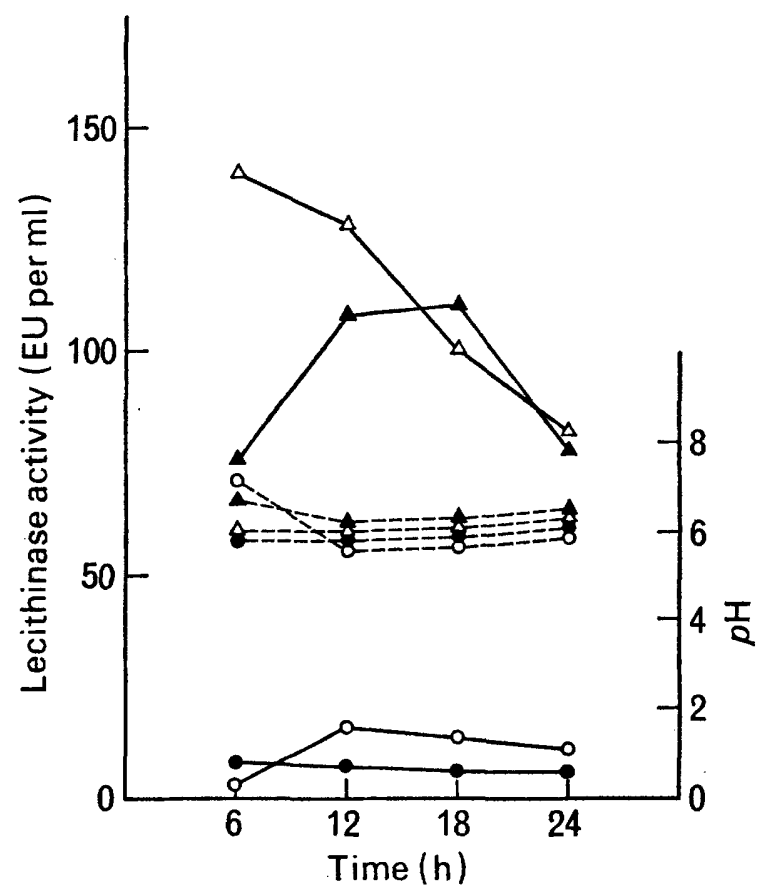

FiG. 2.-Lecithinase production in TYG medium by types B and D. Type B, strain GR2B (O), type B, strain $990(\Theta)$, type $D$, strain $4507(\triangle)$, type D, strain $6700(\Delta) .-=$ Lecithinase activity (EU per $\mathrm{ml})$; $-\cdots=p \mathrm{H}$. 
Differentiation by lecithinase activity. The above results confirm that type-A strains can be readily distinguished from strains of types $B$ and $D$; the zones of lecithinase acitvity given by type-D culture products were distinctly larger than those of type B. This suggested that type D might be distinguished from type $B$ on the basis of a certain level of production of the $\beta$-toxin lecithinase. A preliminary test was performed to find a suitable medium for the production of $\beta$-lecithinase. Two type-B strains ( 990 and GR2B) and two type-D strains (6700 and 4507) were examined for their yields of $\beta$-lecithinase in VfG, TYG and T-F medium after incubation for $6,12,18,24$ and $48 \mathrm{~h}$. The results summarised in fig. 2 show that the yield of $\beta$-lecithinase was highest in the late logarithmic-growth phase which was usually at 6-12 h. The $\beta$-lecithinase produced in the T-F medium was rapidly inactivated during cultivation, whereas the yield remained stable in the TYG and VfG media. No significant changes in $p \mathrm{H}$ occurred in any of the cultures during the 24-48-h incubation period. As the VfG medium gave less consistent and lower yields, the TYG medium was used throughout our experiments. The results of studies of $\beta$-lecithinase production of 15 type-B, 13 type-D and 3 type-B or type-D strains grown in TYG medium for 6 and $12 \mathrm{~h}$ are summarised in table I. The experiment was repeated three times for each strain with different batches of the TYG medium. It is clear that strains of type D produced much more $\beta$-lecithinase than did those of type B and that $20 \mathrm{EU}$ per $\mathrm{ml}$ may be a critical level for differentiation; on that basis, only one or two of our strains appear to be borderline. Lecithinase activities of "non-toxigenic" strains of $C$. oedematiens type B or D did not exceed 2.7 EU per ml throughout these experiments.

TABLE I

Lecithinase activities of Clostridium oedematiens types $B$ and $D$

\begin{tabular}{|c|c|c|c|c|c|c|c|c|}
\hline \multirow{2}{*}{$\begin{array}{c}\text { Type-B } \\
\text { strain } \\
\text { designation }\end{array}$} & \multicolumn{2}{|c|}{$\begin{array}{l}\text { Lecithinase activity } \\
\text { (EU per ml) }\end{array}$} & \multirow{2}{*}{$\begin{array}{l}\text { Type-D } \\
\text { strain } \\
\text { designation }\end{array}$} & \multicolumn{2}{|c|}{$\begin{array}{l}\text { Lecithinase activity } \\
\text { (EU per ml) }\end{array}$} & \multirow{2}{*}{$\begin{array}{c}\text { Type-B } \\
\text { or }-D \\
\text { strain } \\
\text { designation }\end{array}$} & \multicolumn{2}{|c|}{$\begin{array}{l}\text { Lecithinase activity } \\
\text { (EU per ml) }\end{array}$} \\
\hline & minimum & maximum & & minimum & maximum & & minimum & maximum \\
\hline $\begin{array}{l}2879 \\
\text { Kitamura } \\
\text { GR4B } \\
\text { 25758 } \\
\text { GR3B } \\
\text { GR1B } \\
\text { GR11B } \\
\text { GR13B } \\
\text { GR7B } \\
\text { 992 } \\
\text { 990 } \\
\text { GR8B } \\
\text { GR12B } \\
\text { GR2B } \\
\text { 1787 }\end{array}$ & $\begin{array}{l}0 \\
0 \\
0 \\
0 \cdot 1 \\
0 \cdot 8 \\
0 \cdot 9 \\
1 \cdot 3 \\
1 \cdot 4 \\
2 \cdot 1 \\
2 \cdot 7 \\
3 \cdot 8 \\
4 \cdot 5 \\
5 \cdot 1 \\
6 \cdot 9 \\
10 \cdot 1\end{array}$ & $\begin{array}{r}1.1 \\
3.2 \\
7.6 \\
1.1 \\
5.7 \\
1.4 \\
2.9 \\
8.0 \\
10.8 \\
9.7 \\
5.7 \\
8.9 \\
14.3 \\
19.4 \\
14.0\end{array}$ & $\begin{array}{c}\text { Shinketsu } \\
6705 \\
2014 \\
6514 \\
13135 \\
6700 \\
9192 \\
8933 \\
9502 \\
2168 \\
2116 \\
3630 \\
4507\end{array}$ & $\begin{array}{l}10 \cdot 0 \\
21 \cdot 3 \\
23 \cdot 8 \\
24.4 \\
26 \cdot 5 \\
33 \cdot 6 \\
39 \cdot 2 \\
43 \cdot 2 \\
44 \cdot 3 \\
46 \cdot 5 \\
48 \cdot 0 \\
56 \cdot 0 \\
84 \cdot 3\end{array}$ & $\begin{array}{r}15 \cdot 8 \\
64 \cdot 0 \\
43 \cdot 4 \\
35 \cdot 7 \\
38 \cdot 4 \\
117 \cdot 1 \\
182.9 \\
56 \cdot 0 \\
91 \cdot 5 \\
69 \cdot 5 \\
113.4 \\
81 \cdot 2 \\
145.6\end{array}$ & $\begin{array}{l}\text { Non-toxic } \\
\text { Higumakan } \\
\text { Mizuno-81 }\end{array}$ & $\begin{array}{l}0 \\
0 \\
0 \cdot 1\end{array}$ & $\begin{array}{l}0 \cdot 3 \\
2 \cdot 5 \\
2 \cdot 7\end{array}$ \\
\hline
\end{tabular}

$\mathrm{EU}=$ egg-units. 


\section{CLOSTRIDIUM OEDEMATIENS TYPES B AND D}

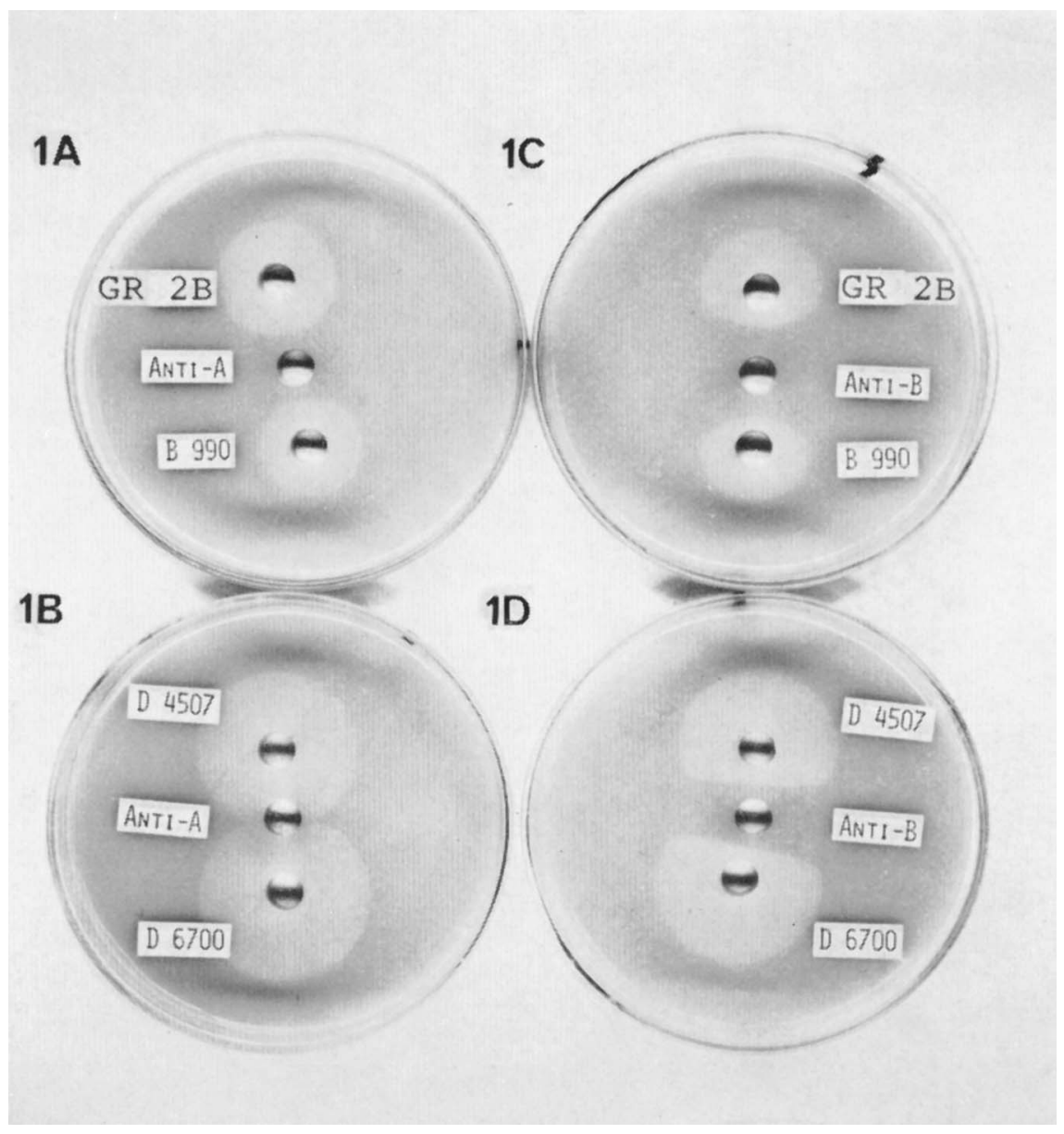

FIG. 1.-Neutralisation tests of lecithinase on egg-yolk plates. Lecithinase reactions produced by samples of culture supernates-in the outer wells-of type-B strains GR2B and 990, and of type-D strains 4507 and 6700 , are not inhibited by anti-A serum in the centre well ( $1 a$ and $1 b$ ) but inhibited by anti-B serum in the centre well $(1 c$ and $1 d)$. 
Studies with mitomycin $C$

All of the strains were examined for susceptibility to MC. The results summarised in table II show that 12 of 13 type-D strains were sensitive to MC at a concentration of 1 or $2 \mu \mathrm{g}$ per $\mathrm{ml}$ of the culture medium. A representative plot of the lytic effect induced by MC is shown in fig. 3(a). Of 15 type-B strains used, however, 14 were insensitive (table II, fig. $3(b)$ ). In addition, 10 type-A strains were tested for susceptibility to MC. Only one, a highly toxigenic stock strain (No. 140), was sensitive.

\section{Demonstration of phage particles}

Attempts were made to demonstrate phage particles in the lysates of the MC-sensitive strains 8933, 4507, 6700, 9502, 9192, and 2014. Phage particles

TABLE II

Biochemical properties and susceptibility to mitomycin $C(M C)$ of $C$. oedematiens types $B$ and $D$

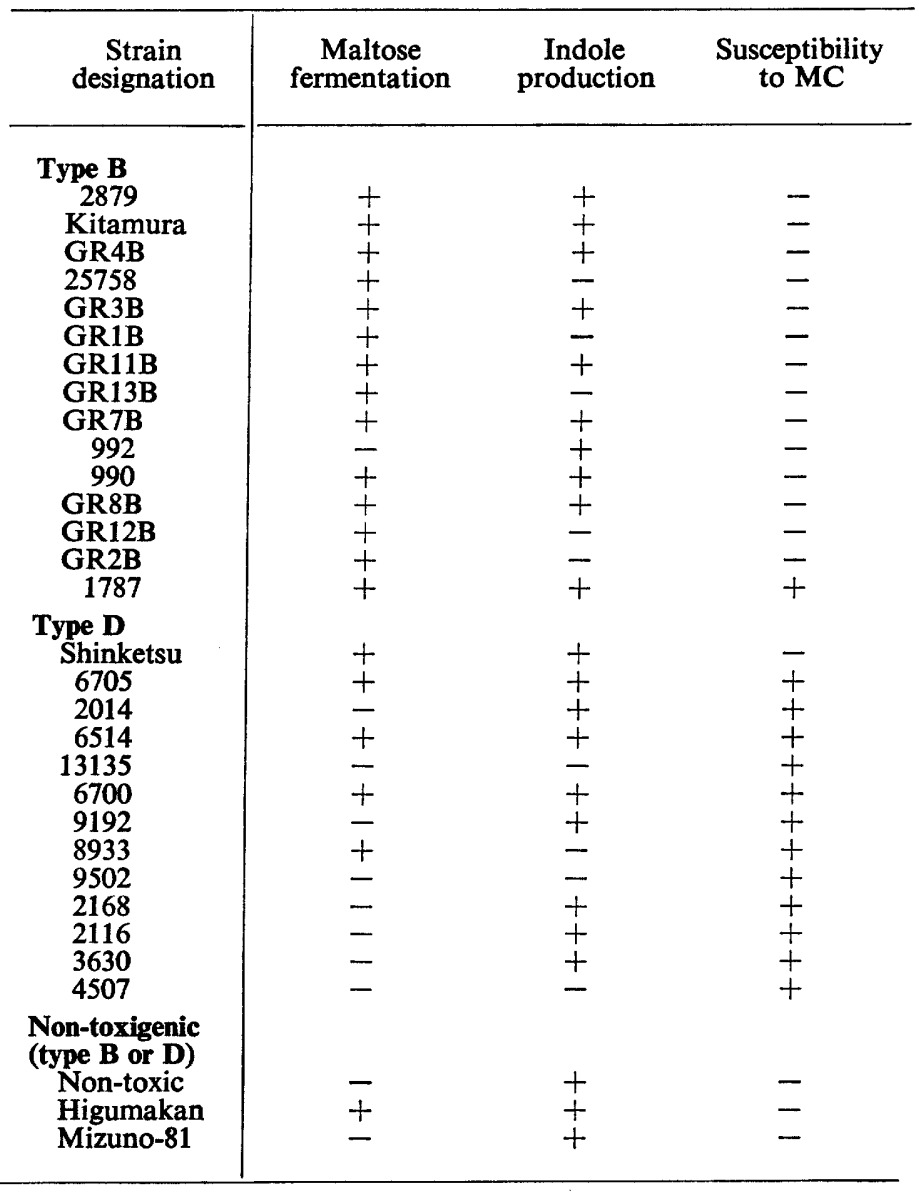

For definitions of positive and negative results see Materials and Methods. J. MED. MICROBIOL.-VOL. 8 (1975) 

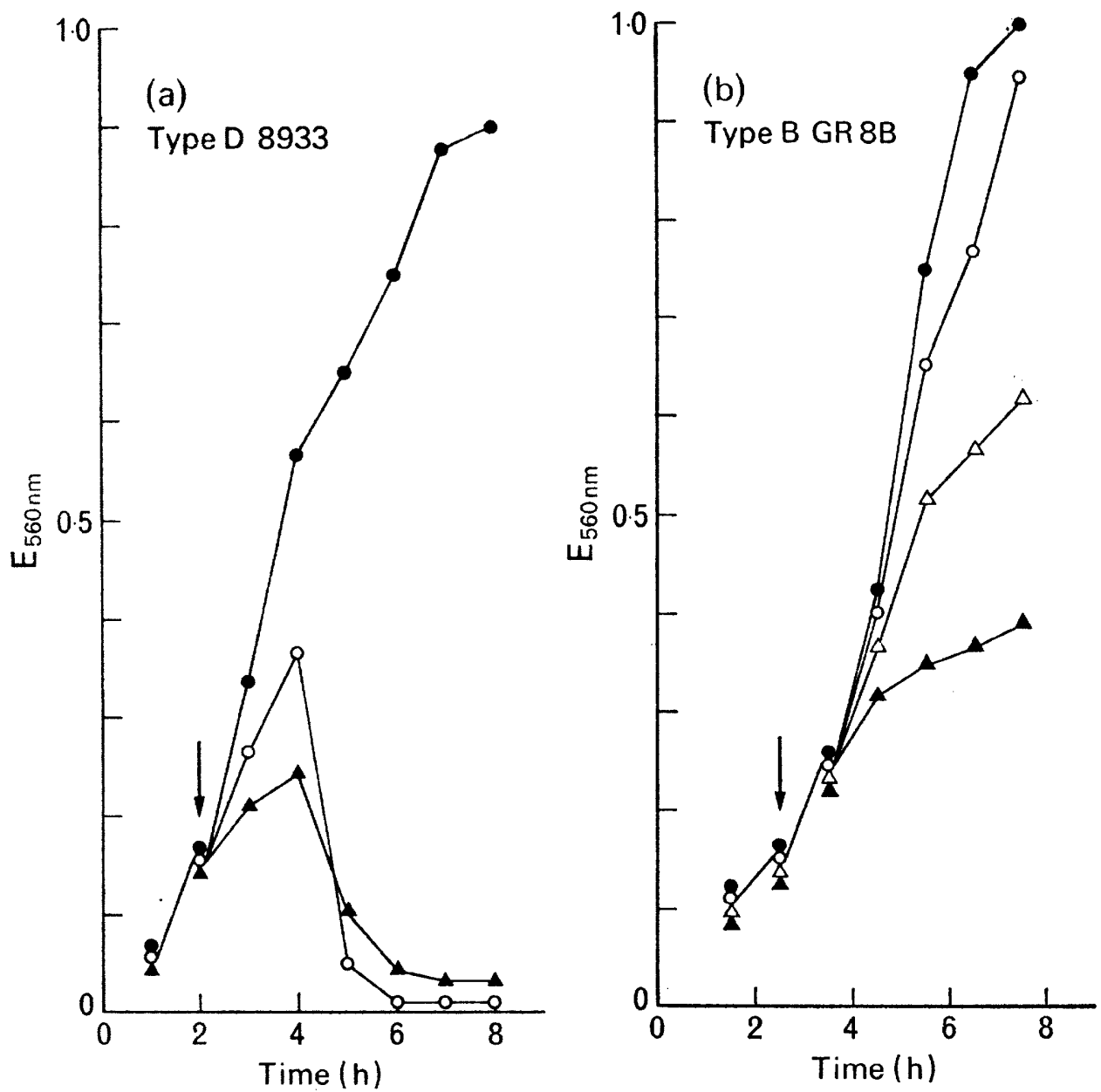

FIG. 3. -The effect of mitomycin C (MC) on Clostridium oedematiens; the mitomycin was added to a suspension of the test organism in culture medium at the time indicated by the arrow in each case (see Methods) and turbidity readings were recorded. Graph $a$ shows the lytic effect obtained with a type-D strain. Graph $b$ shows the lack of lysis obtained with a type-B strain. O-O, MC content, $1.0 \mu \mathrm{g}$ per ml; $\triangle \longrightarrow \triangle$, MC content, $2.0 \mu \mathrm{g}$ per ml; $\Delta-\Delta$, MC content, $5.0 \mu \mathrm{g}$ per $\mathrm{ml}$; $\longrightarrow$, control (no addition of MC).

were observed in deposits from MC-induced lysates of strains 8933,4507 , and 9502 after centrifugation at $100,000 \mathrm{~g}$ for $90 \mathrm{~min}$., but they were not observed in pellets obtained from other strains.

A crude lysate of strain 4507 which showed most phage particles was plated according to the double-agar-layer technique (Adams, 1959) on plates of TYG agar seeded with each of several toxigenic and non-toxigenic strains of type B or D in the top layer, but attempts to demonstrate plaque production were unsuccessful. 
Other tests to differentiate types $B$ and $D$

Indole production provides no basis for differentiation of these types, and the results of maltose fermentation tests produce a less clear-cut differentiation than we obtained with the $\beta$-lecithinase potency tests and the MC susceptibility studies (table II).

\section{DisCUSSION}

Rutter (1970) recommended the use of cooked-meat broth for the study of the carbohydrate-fermentation reactions of $C$. oedematiens; he demonstrated that all of a variety of strains of $C$. oedematiens type B fermented maltose, whereas none of 3 type-D strains fermented the sugar. We found one type-B strain that does not ferment maltose and several type-D strains that are maltose positive. Roberts, Güven and Worrall (1970) did not recognise maltose fermentation as useful for type differentiation. Smith and Holdeman (1968) stated that type-D strains can be distinguished from type-B on the basis of indole production. We tested type-B and type-D strains for the ability to produce indole in cooked-meat broth containing tryptophan $1 \mu \mathrm{g}$ per $\mathrm{ml}$ and we found no type-specific pattern in the results at $2-4$ days.

On the basis of the finding that 10 units of $\beta$-antitoxin were sufficient to suppress the lecithinase or haemolytic activities of $\beta$-toxin produced by type-B strains of $C$. oedematiens, but not enough to suppress those of type-D strains, Oakley and Warrack (1959) and Rutter and Collee (1969) recommended neutralisation tests for the in-vitro typing of this organism. It is, however, not possible to perform meaningful neutralisation tests without accurate information on the antitoxin, as Rutter and Collee (1969) stressed. At present, the $\beta$-antitoxin potency of commercially available antitoxic sera is not generally stated. We therefore estimated lecithinase activity directly according to the method of van Heyningen (1941). Although a distinct difference in lecithinase potency could be found between strains of types $B$ and $D$, further studies with more strains of types $B$ and $D$ are necessary to establish a critical level for differentiation. From our finding that a partly purified preparation of $\beta$ lecithinase of a $C$. oedematiens type-D strain 4507 showed 1441 EU and 396 MLD per $\mathrm{mg}$ of protein respectively, it is manifest that one EU of $\beta$-lecithinase activity of $C$. oedematiens is several times less lethal than one EU of the $\alpha$-toxin lecithinase of $C$. perfringens.

The classical distinction between type-B and type-D strains of $C$. oedematiens rests upon the demonstration of the lethal $\alpha$-antigen in type-B culture products. These tests require laboratory animals. Although Rutter and Collee (1969) showed that $\alpha$-toxin could be demonstrated by cytopathogenic effects in tissue cultures, most laboratories would welcome a test that differentiates between types $B$ and $D$ and avoids the use of animals or tissue cultures and overcomes the difficulties of ill-defined antitoxin potencies. Our practical approach to differentiation of types B and D is as follows. (1) First, demonstration of the $\beta$-lecithinase by a simple plate method. (2) Differentiation by a critical level of $\beta$-lecithinase activity into type B or D. (3) Test for susceptibility to $\mathrm{MC}$. 
Susceptibility of other clostridia, namely C. botulinum to mitomycin C (Kiritani et al., 1973) and of C. sordellii to the agent (Shimamura et al., 1974), has been described already. In the present study, $C$. oedematiens type-D strains were found to be susceptible to MC and phage-like particles were demonstrated in the lysates. The number of the phage-like particles, however,was very low in spite of the abrupt lysis induced by MC. The rapid lysis may be partly or mainly due to autolytic systems inherent in spore-bearing organisms (Altenbern and Stull, 1965; Galli and Hughes, 1965; Kawata and Takumi, 1971) rather than due to the direct effects of phage multiplication.

Although we could not demonstrate plaque-forming phage in the lysate, we frequently observed complete suppression of growth of indicator cells in a soft-agar layer containing an MC-lysate of strain 4507. This suggests that a lytic agent or agents may exist in the MC-lysate, as has been demonstrated with other clostridia (Mitsui et al., 1973; Shimamura et al., 1974).

Our present finding suggests that the susceptibility of $C$. oedematiens to MC may be correlated with $\beta$-toxigenicity. The only MC-insensitive type-D strain (Shinketsu) showed the lowest $\beta$-toxigenicity among the 13 type-D strains tested, whilst the only MC-sensitive type-B strain (1787) showed the highest $\beta$-toxigenicity among 15 type-B strains tested. In the course of the study, however, we found that the $\beta$-toxigenicity of two type-D strains was markedly attenuated or was nearly lost during subculture, although these strains were still MC-sensitive.

\section{SUMMARY}

In tests with broth-culture products of Clostridium oedematiens, none of 15 type-B strains showed $\beta$-toxin lecithinase activities exceeding 20 egg-units per $\mathrm{ml}$, whereas 12 of 13 type-D strains consistently produced much greater amounts of the lecithinase.

The types also differed in their susceptibility to lysis by mitomycin $\mathrm{C}(\mathrm{MC})$. Of 13 type-D strains tested, 12 were sensitive to $\mathrm{MC}$ at a concentration of 1 or $2 \mu \mathrm{g}$ per $\mathrm{ml}$, whilst 14 of 15 type-B strains were insensitive. Phage-like particles were observed in the MC-lysates of some type-D strains. No typespecific differences in the production of indole or the fermentation of maltose were demonstrated.

We thank Professor J. G. Collee for valuable comment and for providing strains of C. oedematiens, Dr Irene Batty, Dr P. Högh, Dr R. Azuma and Dr M. Miyazaki for strains of $C$. oedematiens, Dr K. Mitsui for a supply of a standard $C$. perfringens lecithinase preparation, and $\mathrm{Mr} \mathrm{M}$. Ito for help with the electron microscopy.

\section{REFERENCES}

ADAMS, M. H. 1959. Bacteriophages, London, p. 450.

Altenbern, R. A. ANd Stuld, H. B. 1965. Inducible lytic systems in the genus Bacillus. J. gen. Microbiol., 39, 53.

Galli, E. AND Hughes, D. E. 1965. The autolysis of Clostridium sporogenes. J. gen. Microbiol., 39, 345.

Herningen, W. E. van. 1941. The biochemistry of the gas gangrene toxins. I. Estimation of $\alpha$-toxin of $\mathrm{Cl}$. welchii type A. Biochem. J., 35, 1246. 
KaWATA, T. AND TAKum, K. 1971. Autolytic enzyme system of Clostridium botulinum. 1. Partial purification and characterisation of an autolysin of $C$. botulinum type A. Jap. J. Microbiol., 15, 1.

Kiritani, K., Mitsui, N., Nakamura, S. AND Nishida, S. 1973. Numerical taxonomy of Clostridium botulinum and Clostridium sporogenes strains, and their susceptibilities to induced lysins and to mitomycin C. Jap. J. Microbiol., 17, 361.

Mitsui, K., MrtsuI, N. AND Hase, J. 1973. Clostridium perfringens exotoxins. I. Purification and properties of $\alpha$-toxin. Jap. J. exp. Med., 43, 65 .

NAGLER, F. P. O. 1944. Bacteriological diagnosis of gas gangrene due to Clostridium oedematiens. Nature, Lond., 153, 496.

NishidA, S. AND NAKAGAWARA, G. 1964. Isolation of toxigenic strains of Clostridium novyi from soil. J. Bact., 88, 1636.

OAKLeY, C. L., WarRack, G. H. and Clarke, P. H. 1947. The toxins of Clostridium oedematiens ( $\mathrm{Cl}$. novyi). J. gen. Microbiol., 1, 91 .

OAKLEY, C. L. AND WARRACK, G. H. 1959. The soluble antigens of Clostridium oedematiens type D (Cl. haemolyticum). J. Path. Bact., 78, 543.

Roberts, R. S., GüVEn, S., AND WorRall, E. E. 1970. Studies on Clostridium oedematiens. J. comp. Path. Ther., 80, 9.

RUTTER, J. M. 1970. A study of the carbohydrate-fermentation reactions of Clostridium oedematiens ( $\mathrm{Cl}$. novyi). J. med. Microbiol., 3, 283.

Rutrer, J. M. AND ColleE, J. G. 1969. Studies on the soluble antigens of Clostridium oedematiens (Cl. novyi). J. med. Microbiol., 2, 395.

SMITH, L. DS. AND Holdeman, L. V. 1968. The pathogenic anaerobic bacteria, Illinois, p. 343.

Shimamura, T., Nakamura, S., Hayase, M. and Nishida, S. 1974. Mitomycin-induced lysis of Cl. sordellii. J. med. Microbiol., 7, 277.

TAMAI, K. AND FUKUDA, J. 1970. Studies on anaerobic bacteria in oral cavity. 1. Isolation medium. J. Jap. stomatological Soc., 19, 495 (in Japanese).

WINKLE, S. 1955. Microbiologische und serologische Diagnostik, 2nd ed., Stuttgart, p. 275. 